\title{
Development of paclitaxel loaded pegylated gelatin targeted nanoparticles for improved treatment efficacy in non-small cell lung cancer (NSCLC): an in vitro and in vivo evaluation study
}

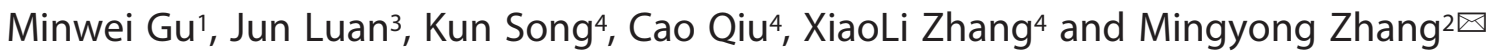 \\ 1Department of Thoracic, Affiliated Hospital of Jiang Nan University, WuXi214062, China; 2Department of Thoracic Surgery, Qing Dao Municipal \\ Hospital, Qing Dao, 266011, China; '3Centre of Animal, Plant and Food testing, Nan Jing customs District P. R. China, Nan Jing, 210001, China; \\ ${ }^{4}$ Nanjing Hope Medical Laboratory Co., Ltd, Nan Jing, 210001, China
}

Purpose: To develop and evaluate paclitaxel (PTX) loaded pegylated gelatin targeted nanoparticles for improved efficacy in non-small cell lung cancer (NSCLC) treatment. Method: PTX loaded gelatin nanoparticles (PTX-GNP) were prepared by crosslinking with glutaraldehyde aqueous solution. These nanoparticles (NPs) were further incubated with PEG 400 to form PEGylated NPs (PEG-PTX-GNP). The NPs were evaluated for surface morphology, size, zeta potential, encapsulation efficiency, drug loading, in vitro drug release, cytotoxicity in an assay on cancer cell lines L132, in vitro cellular uptake in an assay in L132 and 293T cell lines, in vivo antitumor activity on female Balb/c mice, pulmonary deposition, histopathology, and immunohistochemical properties. Results: The nanoparticles were of spherical shape with smooth surface characteristics. The observed DL was of 20.18 to $32.11 \%$, as particle size was of 90 to $115 \mathrm{~nm}$. Zeta potential and polydispersity index (PDI) were within acceptable ranges. Encapsulation was effective when the NPs had a size of $80.50 \mathrm{~nm}$ to $98.12 \mathrm{~nm}$. The PEGylated PTX loaded nanoparticles (PEG-PTX-GNP, GNP4) showed similar PTX release profile to that of the NP4 formulation. PEGylated NPs showed the desired PTX release pattern that is required for cancer treatment. In an in vitro cytotoxicity study, PEG-PTX-GNP showed the maximum antiproliferative activity over the period of 24 hours, followed by PTX-GNP, pure PTX and BPEG-GNP. PEG-PTXGNP showed the highest internalization within both cell lines, followed by PTX-GNP and pure PTX. The survival rate of animals in PEG-PTX-GNP group was $100 \%$, proving the safety and efficacy of the treatment. PEG-PTXGNP showed the highest antitumor activity as compared to other formulations. The pulmonary deposition rate was the highest (6.5 to $12.55 \mu \mathrm{g} / \mathrm{g}$ ) in PEG-PTX-GNP formulations. Histopathology and immunohistochemical study proved that PEG-PTX-GNP had greater anticancer potential than other tested formulations. Conclusion: This study confirms the potential use of paclitaxel loaded PEGylated gelatin targeted nanoparticles for improved efficacy in non-small cell lung cancer (NSCLC) treatment.

Keywords: antitumor, gelatin, nanoparticles, non-small cell lung cancer, Paclitaxel

Received: 21 July, 2020; revised: 05 April, 2021; accepted: 28 April, 2021; available on-line: 06 August, 2021

凶e-mail: mingyong2020@sina.com

Abbreviations: EPR, enhanced permeation and retention; IPA, isopropyl alcohol; HCPT, 10-hydroxycamptothecin; NSCLC, non-small cell lung cancer; PEG, polyethylene glycol; PEG-PTX-GNP, PEGyla- tion of PTX loaded gelatin nanoparticles; PTX, paclitaxel; PTX-GNP, PTX loaded gelatin nanoparticles; SCLC, small cell lung carcinoma

\section{INTRODUCTION}

The lung cancer, also known as pulmonary carcino$\mathrm{ma}$, is a malignant tumor characterized by uncontrolled growth of the cells in pulmonary tissues. Each year, nearly one million people are diagnosed with lung cancer worldwide and nearly $25 \%$ of deaths are caused by cancer, among which pulmonary cancer is the major cause of mortality (Kim et al., 2012). Smoking, alcohol consumption, high fat diet and obesity are the main risk factors for the development of lung cancer in patients. The WHO has already given serious alarm regarding severe rise in mortality due to smoking and unhealthy diet in industrial countries (Torre et al., 2016). The lung cancer is categorized in two main types: small cell lung carcinoma (SCLC) and non-small cell lung carcinoma (NSCLC). In one survey conducted in the USA it was found that among 220,000 lung patients, $85 \%$ of the early cases were NSCLC and the remaining were SCLC. In the current scenario, the available options for the treatment of NSCLC are chemotherapy, surgery, radiation therapy or combination of these (Pless et al., 2015). In the advanced stage of NSCLC, chemotherapy is found ineffective or toxic to the patients. The remaining treatment options have a very low success rate and are systemically toxic due to non-specific effects, which kill normal cells (Garon et al., 2015). The limitations of conventional drug delivery systems prompted the development of alternative approaches to overcome issues such as systemic toxicity. A combination of the anticancer drug with a natural bioactive molecule may be one of the solutions to reduce the side effects. Another approach is to use a nano size carrier system to increase the therapeutic concentration of a drug in a cancer cell due to enhanced permeation and retention (EPR). In addition to the microspheres, liposomes, and lipidic nanoparticles, the polymeric nanoparticles have been extensively used for targeted and controlled drug delivery. Among these systems, nanoparticles have gained prominent attention in the treatment of various types of cancer, including lung cancer. The surfaces of the nanoparticles can be decorated or modified to better target cancer cells that the immune system cannot identify. Nanoparticles can be easily distributed in the tumor area due to the excessive permeability of the tumor vasculature, lack of lymphatic drainage, and the EPR effect. The drug concentration in nanoparticulate form can be increased 10 to 100 times compared to the 
free drug (Scott et al., 2007; Ahmad et al., 193; Peng et al., 2009; Tahara et al., 2017).

Many anti-cancer drugs have been studied in recent years, but most of them are hydrophobic and poorly soluble. Paclitaxel (PTX) is also a potent anti-cancer drug widely used in the treatment of NSCLC, but the clinical use of PTX is limited by high toxicity and low bioavailability due to poor solubility. Taxol is a preparation containing PTX used in the treatment of NSCLC, but it has been associated with various side effects such as cardiotoxicity, nephrotoxicity, neurotoxicity, and hypersensitivity. It has also been observed that PTX concentration in lung tissue is too low to elicit the therapeutic effect in treatment of the NSCLC. Thus, considering all these drawbacks of the current drug delivery system, there is a strong need to reduce side effects and increase the therapeutic efficacy of PTX (Su et al., 2012; Shenb et al., 2012; Goncalves et al., 2001).

Gelatin is a naturally occurring protein biopolymer that is obtained from collagen under acidic or alkaline treatment. Having low antigenicity, it is widely used in parenteral formulations and approved as a plasma expander. In addition, gelatin has many advantages: it is biodegradable, biocompatible, non-toxic, water soluble, nonpyrogenic, inexpensive, can be easily sterilized, and has a higher content of amino acids such as glycine, proline, and alanine which stabilize the triple helical structure of gelatin (Tseng et al., 2008). Gelatin can be easily chemically modified and conjugated due to the presence of ionizable groups such as amine, phenol, carboxyl, guanidine, and imidazole. Glutaraldehyde-crosslinked gelatin has greater stability, and increased circulation time in vivo compared to unmodified gelatin. All these properties make gelatin an ideal candidate for nanoparticulate drug delivery system (Karthikeyan et al., 2013; Lu et al., 2004).

Recently, particular attention has been paid to use of surface-modified gelatin nanoparticles in the treatment of cancer. Surface modification of gelatin nanoparticles with polyethylene glycol (PEG) has been widely used to prepare long circulating nanoparticles. PEGylated nanoparticles were shown to be less prone to degradation in calf serum than non-PEGylated nanoparticles (Hao et al., 2012). It was also observed that the particle accumulation in tumor was nearly 6-fold higher for PEGylated nanoparticles, as compared to non-PEGylated nanoparticles (Schädlich A et al., 2015). Doxorubicin-loaded PEGylated chitosan nanoparticles were found to be more efficient in inhibiting tumor growth than free doxorubicin and non-PEGylated doxorubicin-loaded nanoparticles (Scheeren et al. 2016). Another study showed that co-delivery of doxorubicin and paclitaxel in PEGylated nanoparticles was more efficient in the treatment of non-small cell lung cancer than using non-PEGylated nanoparticles for drug delivery ( $\mathrm{Lv}$ et al., 2016). In a similar study, folic acid conjugated PEG nanoparticles were used for lung cancer targeting in mice model, where they showed enhanced cellular uptake of the nanoparticles confirmed by optical imaging due to receptor-mediated endocytosis (Yoo et al., 2012). Another study developed PEGylated lipid nanocarriers to improve the anti-tumor activity of 10-hydroxycamptothecin (HCPT) in the treatment of lung cancer. An in vivo study showed that the developed PEGylated lipid nanoparticles had better efficacy against A549 lung cancer compared to the HCPT solution and non-PEGylated lipid nanocarriers (Zhang et al., 2008). In this research, we developed surface-modified PEGylated PTX gelatin nanoparticles for the treatment of NSCLC that would reduce side effects, cytotoxicity, increase cellular uptake, and improve biodistribution in lung cancer tissue.

\section{MATERIALS AND METHODS}

\section{Materials}

Paclitaxel (PTX) was purchased from Baoji Guokang Bio-Technology Co., Ltd. (Baoji, Shaanxi, China); Gelatin Type-B, $175 \mathrm{~g}$ Bloom (from bovine skin) was purchased from Sigma-Aldrich (USA); Tween 80, sodium sulfate, isopropyl alcohol, glutaraldehyde (25\%), sodium metabisulfite solution (12\%), Sephadex G-50 column, and polyethylene glycol 400 (PEG 400) were purchased from Sigma-Aldrich USA.

Cell culture and animals. The NSCLC cell lines (293T and L132) for this study were obtained from the Shanghai Institute of Cell Biology. The cells were cultured in Roswell Park Memorial Institute 1640 (RPMI1640) medium supplemented with $10 \%$ fetal bovine serum, $1 \%$ penicillin and $100 \mu \mathrm{g} / \mathrm{ml}$ streptomycin sulphate at $37^{\circ} \mathrm{C}$ in atmosphere of $5 \% \quad \mathrm{CO}_{2} . \mathrm{BALB} / \mathrm{c}$-nu/nu athymic mice were obtained from the Centre of Animal, Plant and Food Testing, Nan Jing customs District. P.R. China, Nan Jing, 210001, China. All animal experiments were conducted with accordance with institutional animal ethical committee of Centre of Animal, Plant and Food Testing, Nan Jing customs District. P.R. China, Nan Jing, 210001, China, with prior approval (ETHL/00432/ CAPFT/2019/01)

Preparation of PTX loaded gelatin nanoparticles. PTX loaded gelatin nanoparticles were prepared using $175 \mathrm{~g}$ Bloom gelatin, which indicates the molecular weight of the gelatin polymer. Gelatin was dissolved in

Table 1. Composition of PTX loaded gelatin nanoparticles (PTX-GNP)

\begin{tabular}{|c|c|c|c|c|c|}
\hline Sr. No & Ingredients & NP1 & NP2 & NP3 & NP4 \\
\hline 1 & PTX (mg) & 2 & 2 & 2 & 2 \\
\hline 2 & Gelatin (mg) & 100 & 150 & 200 & 250 \\
\hline 3 & Tween 80 (mL) & 5 & 10 & 15 & 20 \\
\hline 4 & $20 \%$ Sodium sulfate aqs solution $(\mathrm{mL})$ & 1.5 & 1.5 & 2.0 & 2.0 \\
\hline 5 & Isopropyl alcohol (mL) & 1 & 1 & 1 & 1 \\
\hline 6 & Double distilled water $(\mathrm{mL})$ & q.s. & q.s. & q.s. & q.s. \\
\hline 7 & $20 \%$ Sodium sulfate aqs solution $(\mathrm{mL})$ & 5 & 5 & 6 & 6 \\
\hline 8 & $20 \%$ Glutaraldehyde (mL) & 0.5 & 0.5 & 1 & 1.5 \\
\hline 9 & $1.5 \%$ sodium metabisulfite solution $(\mathrm{mL})$ & 4 & 4 & 5 & 5 \\
\hline
\end{tabular}


water containing varying amount of Tween 80 . This polymeric solution was heated at $40-45^{\circ} \mathrm{C}$ under continuous magnetic stirring at $400 \mathrm{rpm}$ for $1 \mathrm{~h}$. Next, 1.5 to $2 \mathrm{~mL}$ of $20 \%$ aqueous solution of sodium sulfate was added drop wise under continuous stirring. PTX was dissolved in isopropyl alcohol (IPA) at a concentration of $2 \mathrm{mg} /$ $\mathrm{mL}$ and added to gelatin solution containing sodium sulfate, then 5 to $6 \mathrm{~mL}$ sodium sulfate solution was added with stirring until the solution became cloudy. The appearance of turbidity indicates the formation of gelatin aggregates. Next, nearly $1 \mathrm{~mL}$ double distilled water was added drop wise to the solution, until it became clear again. An aqueous solution of glutaraldehyde (20\%) was then added to crosslink the gelatin. The crosslinking process was stopped by adding 4 to $5 \mathrm{~mL}$ of sodium metabisulfite solution $(15 \%)$. The resulting solution was stirred for $1 \mathrm{~h}$ and the product was purified on Sephadex G-50 column (Amjadi et al., 2019). The nanoparticles were lyophilized in a freeze dryer for $72 \mathrm{~h}$ and stored at $-20^{\circ} \mathrm{C}$. The composition of the formula is shown in Table 1.

PEGylation of PTX loaded gelatin nanoparticles (PEG-PTX-GNP). PEGylation of PTX-GNP was performed immediately after lyophilization of the nanoparticles. PTX-GNP were incubated with $1500 \mu \mathrm{g}$ of PEG 400 (molecular weight $8000-10,000$ ) per milligram nanoparticle and further diluted with PBS buffer to a final volume of $200 \mu \mathrm{L}$ and incubated for 30 minutes at room temperature using rotary shaker (Amjadi et al., 2019).

\section{Statistical analysis}

Statistical analysis was done using a SAS statistical kit (Version 9.0; SAS Institute, Inc., Cary NC). The differences were considered statistically significant at $p<0.05$.

\section{Characterization of PTX-GNP and PEG-PTX-GNP}

PTX entrapment efficiency in gelatin nanoparticles. The PTX loaded nanoparticulate suspension was centrifuged at $20,000 \mathrm{rpm}$ for $30 \mathrm{~min}$ and the clear supernatant was separated by decantation. The amount of free (unloaded) drug was determined by injecting the supernatant liquid into an HPLC equipped with UV detector. The PTX entrapment efficiency in gelatin nanoparticles was calculated using the following equation (Hamarat et al., 2016):

\section{$\%$ E.E of PTX=Total PTX-free PTX/Total PTX amount} $\times 100$

PTX loading in gelatin nanoparticles. Five milligrams of PTX loaded gelatin nanoparticles was added to hot water and shaken for 1 hour in a water bath shaker maintained at $40-45^{\circ} \mathrm{C}$, to allow dissolving of the outer layer of gelatin. Nearly $15 \mathrm{ml}$ of ethanol was added to the flask with dissolved gelatin. The flask was then shaken by hand for 15 minutes to extract the PTX in ethanol. The dispersion was then filtered through a 0.45 $\mu \mathrm{m}$ syringe filter. The filtrate containing PTX was determined by HPLC. The PTX loading was calculated according to the following formula (Hamarat et al., 2016).

$\%$ PTX loading=PTX Entrapped/Weight of nanoparticles $\times 100$

PTX release from gelatin nanoparticles. PTX loaded gelatin nanoparticles $(20 \mathrm{mg})$ were dispersed in 50 $\mathrm{mL}$ of $\mathrm{pH} 7.4$ phosphate buffer and incubated at $37^{\circ} \mathrm{C}$. Samples $(2 \mathrm{~mL})$ were taken at a predetermined time interval and replenished with fresh buffer solution $(2 \mathrm{~mL})$ to maintain the sinking conditions. The taken sample was centrifuged for 20 minutes at 70,000 rpm using an ultracentrifuge. The nanoparticle-free supernatant was separated and extracted twice with $5 \mathrm{~mL}$ of ethanol. The extracted solution was filtered through a $0.45 \mu \mathrm{m}$ syringe filter and used to estimate PTX release from nanoparticles using HPLC (Hamarat et al., 2016).

Particle size, Zeta potential polydispersity index (PDI) and yield of nanoparticles. PTX loaded gelatin nanoparticles were dispersed in double distilled water (10 $\mathrm{mL}$ ) and sonicated for $5 \mathrm{~min}$ in a water batch sonicator. The nanoparticulate suspension was diluted 10 times with double distilled water and a drop of suspension was mounted on foil paper, dried coated with gold and examined under scanning electron microscopy (SEM) (Leo 435 VP, Cambridge, UK.) at an operating distance of $8-8.5 \mathrm{~mm}$ and accelerating voltage of $15.0 \mathrm{kV}$. Particle size, PDI and zeta potential of diluted nanoparticles were measured using Zetasizer (Malvern instruments DTS Ver 4.10). The yield of nanoparticles was calculated from the weight of freeze dried PTX loaded gelatin nanoparticles.

Cytotoxicity assay study. Cytotoxicity of the nanoparticulate formulations was determined in L132 cancer cell line with a 3-(4,5-dimethylthiazol-2-yl)-2,5 diphenyl tetrazolium bromide assay. The cells were grown in 96 well tissue culture plate at $1 \times 10^{5}$ per well. The cells were treated either with PTX loaded gelatin nanoparticles (PTX-GNP), PTX loaded PEGylated gelatin nanoparticles (PEG-PTX-GNP), pure PTX or blank PEGylated gelatin nanoparticles (BPEG-GNP) at concentrations of $0.5 \mathrm{mg} / \mathrm{mL}$ and $0.75 \mathrm{mg} / \mathrm{mL}$ for 48 hours. 3-(4,5-dimethylthiazol-2-yl)-2,5 diphenyl tetrazolium bromide stock solution was added to each culture at $10 \%$ of the final culture volume and the cells were incubated for 4 hours at $37^{\circ} \mathrm{C}$. Then, the medium was collected, and centrifuged, and dimethyl sulfoxide was added to it to dissolve the formazan crystals. The absorbance of the medium was measured at $490 \mathrm{~nm}$ using microplate reader. The cytotoxicity was calculated as the percentage of viability in each well (Singh et al., 2008).

In vitro cellular uptake assay in L132 and 293T cell lines. A cellular uptake assay was used to determine the accumulation of PTX released from nanoparticles in the cell line. L132 and 293T cell lines were seeded in 12 well culture plates at a density of $10^{5}$ cells/well and incubated for $24 \mathrm{~h}$. Incubated cell lines were treated with either PTX-GNP, PEG-PTX-GNP or pure PTX at concentrations of $0.03,0.06$ and $1.0 \mu \mathrm{M}$ for $8 \mathrm{~h}$. After incubation, the cells were trypsinized and washed with saline buffer three times. The cells were centrifuged, and the cell mass was treated with lysis buffer and sonicated. The resulting solution was analyzed with HPLC to determine the internal accumulation of the drug in the cell lines (Liu et al., 2011).

In vivo antitumor activity. In vivo antitumor activity of PTX-GNP, PEG-PTX-GNP and pure PTX was studied in 8 - to 10 -week-old female Balb/c mice. Each of the animals was anesthetized with isoflurane and had its left flank shaved and their left lung injected at a depth of $4 \mathrm{~mm}$ with approximately 1.4 million L132 cells in exponential growth phase. The animals were kept under observation (nearly 24 days) until the palpable tumors formed. The animals were divided in to four groups as follows: 1) PTX-GNP treated 2) PEG-PTX-GNP treated 3) pure PTX treated 4) no treatment or control group. All formulations were administered by inhalation to each group of animals at a dose of $3 \mathrm{mg} / \mathrm{kg}$ for 3 days using nasal insufflators. The experiment was continued until the established end points, at which the volume and weight of the tumors were measured to determine the 
Table 2. Physical characterization of nanoparticles

\begin{tabular}{|c|c|c|c|c|}
\hline Type of NP & \multicolumn{4}{|c|}{ PTX loaded gelatin NP (PT-GNP) } \\
\hline Batch Code & NP1 & NP2 & NP3 & NP4 \\
\hline Particle size & $90.44 \mathrm{~nm}$ & $96.50 \mathrm{~nm}$ & $101.59 \mathrm{~nm}$ & $110.525 \mathrm{~nm}$ \\
\hline PDI & 0.125 & 0.135 & 0.234 & 0.298 \\
\hline Zeta potential & $19.12 \pm 5.5$ & $20.20 \pm 2.5$ & $21.25 \pm 2.5$ & $22.25 \pm 3.0$ \\
\hline Type of NP & \multicolumn{4}{|c|}{ PEGylated PTX-loaded gelatin NP (PEG-PTX-GNP) } \\
\hline Batch Code & GNP1 & GNP2 & GNP3 & GNP4 \\
\hline Particle size & $93.24 \mathrm{~nm}$ & $97.99 \mathrm{~nm}$ & $103.5 \mathrm{~nm}$ & $112.50 \mathrm{~nm}$ \\
\hline PDI & 0.130 & 0.143 & 0.250 & 0.30 \\
\hline Zeta potential & $20.10 \pm 3.5$ & $20.25 \pm 2.3$ & $21.30 \pm 4.5$ & $22.30 \pm 3.5$ \\
\hline
\end{tabular}

antitumor activity of investigated treatment (Long et al., 2014).

Pulmonary deposition study. The amount of PTX deposited in the lungs of PTX-GNP-, PEG-PTX-GNPor pure PTX-treated animals was assessed at 1, 3, 12 and $24 \mathrm{~h}$ post treatment. The animals were sacrificed, and their lungs were carefully removed, washed thrice with $\mathrm{pH} 7.4$ phosphate buffer and soaked in nitric acid solution $(70 \%)$ for $20 \mathrm{~h}$. The digested and homogenized lungs were centrifuged and the supernatant was HPLCanalyzed to determine the PTX content (Long et al., 2014).

Histopathology study. Histopathology study was performed on separated cancer tissues from animals. The tumors were cut in to $30 \mu \mathrm{m}$ sections, deparaffinized and rehydrated with eosin and hematoxylin. Slides were viewed under a light microscope and pictures were taken (Li et al., 2017).

Immunohistochemical study. The tumor samples were collected and immediately put in $4 \%$ buffered paraformaldehyde for 12 hours. The samples were then rehydrated with alcohol gradient and embedded in paraffin. The paraffin blocks were sliced into $5 \mu \mathrm{m}$ sections and mounted on poly-l-lysine-coated slides. Next, deparaffinized tumor sections were rehydrated with 3\% hydrogen peroxide and alcohol and incubated for $30 \mathrm{~min}$ with capase-3 and MMP-9 antibodies and stained (Li et al., 2017).

\section{RESULTS AND DISCUSSION}

\section{Preparation of PEGylated, PTX-loaded gelatin nanoparticles (PEG-PTX-GNP)}

PEGylated PTX-loaded gelatin nanoparticles were produced in two steps: 1) synthesis of PTX loaded gelatin nanoparticles followed by 2) PEGylation of PTX loaded gelatin nanoparticles. PEGylation of nanoparticles is widely recommended for enhanced biocompatibility, non-immunogenicity, nontoxicity, and low protein adsorption. PEGylation also increases the blood circulation time and cellular uptake of the nanoparticles. The surface characteristics of nanoparticles (PTX-GNP) were examined using FESEM. The nanoparticles had a spherical shape with a smooth surface (Fig. 1). In dry form, the nanoparticles were separated from each other and had free flowing properties. The PEGylation of nanoparticles (PEG-PTX-GNP) did not affect their surface characteristics.

The size, zeta potential and PDI are important factors in determining the stability and performance of an NP. The NP size between 60 and $200 \mathrm{~nm}$ is considered suitable for cancer therapy as it enhances the EPR effect in passive targeting. Up to $80 \%$ of aerosol particles measuring less than $1 \mu \mathrm{m}$ in diameter can be easily exhaled after inspiration, without being deposited. Particles smaller than $100 \mathrm{~nm}$ can effectively deposit in alveolar region leading to improved chemotherapy outcomes for sensitive and resistant non-small cell lung cancer (Mehrotra et al., 2011). Both PTX-GNP and PEG-PTX-GNP NP are of appropriate size to protect against RES by avoiding endocytosis and destruction. Zeta potential values between $-30 \mathrm{mV}$ and $+30 \mathrm{mV}$ are considered ideal for achieving better physical stability of the nanoparticle dispersion and suspension. Both PT-GNP and PEGPTX-GNP had ideal zeta potential values to maintain their stability (Du et al., 2009). PDI of both types of nanoparticles were also found within acceptable range (less than 0.3), which indicated homogeneous dispersion of the NPs. The narrow PDI of nanoparticles may be due to the presence of PEG chains that prevent nanoparticle aggregation. Overall, the prepared nanoparticles had improved stability, PDI, zeta potential and particle size. Table 2 shows all physical parameters of the NPs.

The EE of PTX in nanoparticles was determined by calculating the free (non-entrapped) drug content in supernatant using HPLC. The EE of all formulations was between 80.50 and $98.12 \%$. A direct relationship was observed between the concentration of gelatin and EE. In this study, we have used gelatin Type-B, $175 \mathrm{~g}$ Bloom . Gelatin, due to its gelling and sticky properties, has sufficient strength to hold the drug inside the nanoparticles, which increased the EE and DL of PTX (Karthikeyan et al., 2013). The observed DL within nanoparticle for-

Table 3. PTX entrapment efficiency and loading in gelatin nanoparticles

\begin{tabular}{lllll}
\hline Type of NP & \multicolumn{2}{l}{ PTX loaded gelatin NP (PT-GNP) } & & NP3 \\
\hline Batch Code & NP1 & NP2 & NP4 \\
\hline$\%$ EE & $80.50 \mathrm{~nm}$ & $82.90 \mathrm{~nm}$ & $97.89 \mathrm{~nm}$ & $98.12 \mathrm{~nm}$ \\
\hline$\%$ DL & 20.18 & 27.12 & 30.17 & 32.11 \\
\hline$\%$ Yield & 78.20 & 82.25 & 90.25 & 95.12 \\
\hline
\end{tabular}




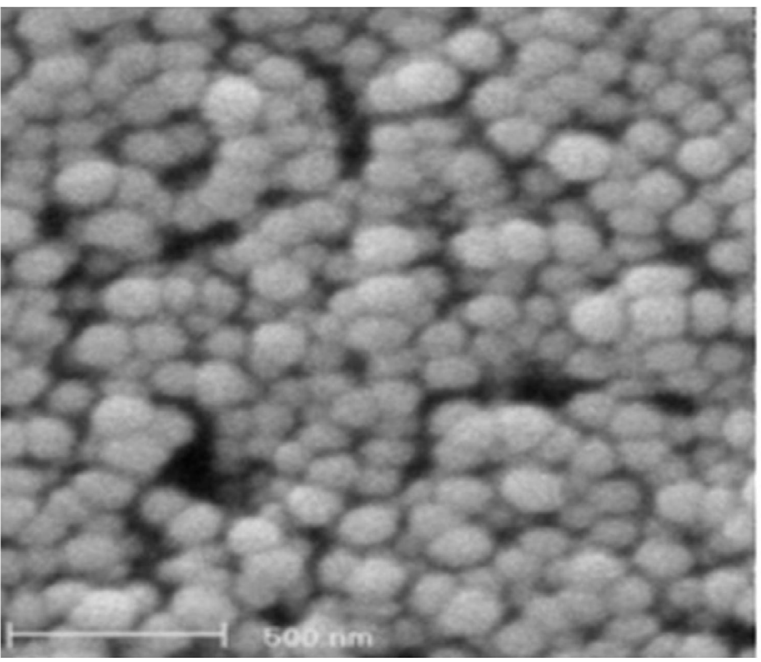

Figure 1. Surface morphology of PEGylated NPs showing spherical and smooth surface characteristics

mulations was between 20.18 and $32.11 \%$. The yield of NPs ranged from $78.20 \%$ to $95.12 \%$ and increased with increasing concentration of gelatin. Table 3 shows comparative EE, DL and yield of NP's.

\section{PTX release from PTX-GNP and PEG-PTX-GNP}

In vitro PTX release form PTX-GNP and PEG-PTXGNP was studied in $\mathrm{pH} 7.4$ saline phosphate buffer. NP4 formulation showed initial burst release of $20.13 \%$ from PTX-GNP nanoparticles followed by a sustained release of almost $67 \%$ over a period of 40 hours. The slow and gradual release was observed due to the greater amount of gelatin used in the nanoparticle preparation as compared to other formulations such as NP1, NP2 and NP3. Formulations containing lower amount of gelatin did not show satisfactory PTX release profile as shown in Fig. 2.

The PEGylated PTX-loaded nanoparticles (PEG-PTXGNP, GNP4) showed similar PTX release profile to that of NP4 formulation (Fig. 2). This observation clearly indicated that PEGylation of nanoparticles did not alter the release characteristics of the pure PTX loaded gelatin nanoparticles. Sustained and gradual release of a drug is always a desirable feature in cancer treatment to provide a longer duration of therapeutic action. PEGylated NPs

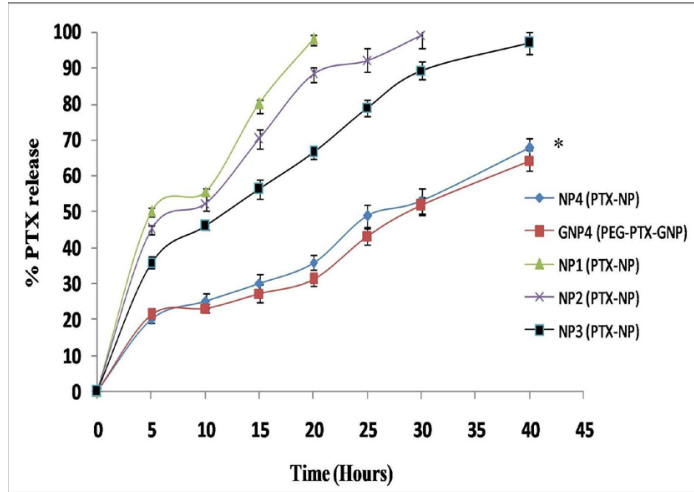

Figure 2. Comparative in vitro PTX release profile from PEGylated and non-PEGylated NPs in pH 7.4 saline phosphate buffer. The nanoparticulate formulation showed a sustained release behavior and the release was significantly prolonged $\left({ }^{*} P<0.05\right.$.) Data presentation of mean S.D. of \pm 0.5 . showed a desired PTX release pattern that is required for the cancer treatment.

\section{Cytotoxicity assay study}

The cytotoxicity of pure PTX, blank PEGylated gelatin nanoparticles (BPEG-GNP), PTX-GNP and PEGPTX-GNP were determined in the human SCLC cell line L132. All formulations were incubated with the cells for the period of 24 hours at two different concentrations. Cytotoxicity was calculated based on cell viability. Among all these formulations, PEG-PTX-GNP showed the maximum antiproliferative activity (i.e., cytotoxicity) over the period of 24 hours, followed by PTX-GNP, pure PTX and BPEG-GNP. The cytotoxicity was concentration dependent i.e., the higher the concentration, the higher the cytotoxicity. PEG-PTX-GNP (30\%, $25.5 \%$ cell viability) showed nearly two-fold increase in cytotoxicity as compared to PTX-GNP (55.9\%, 50.6\% cell viability), as shown in Fig. 3 .

PTX interferes with microtubule growth, which destroys the cell's cytoskeleton and shows the antitumor activity (Tran et al., 2014). In our experiments, pure PTX showed cytotoxic activity when compared to blank PEGylated gelatin nanoparticles (BPEG-GNP). After phagocytosis or endocytosis, the nanoparticles reach the acidic lysosomes, and if they contain the drug, its release takes place in a sustained and gradual manner.

\section{In vitro cellular uptake assay in L132 and 293T cell lines}

Cellular uptake assay was conducted in L132 and $293 \mathrm{~T}$ cell lines over the period of $12 \mathrm{~h}$ at various NP concentrations, ranging from 0.03 to $1.0 \mu \mathrm{M}$. A linear correlation was observed between the NPs' concentration and cellular uptake in both cell lines. In both cell lines, PEG-PTX-GNP showed the highest internalization, followed by PTX-GNP and pure PTX. PEG-PTXGNP showed nearly two-fold higher cellular uptake as compared to PTX-GNP, as shown in Fig. 4.

The PEGylation of nanoparticles protects them from opsonization, surface aggregation, and phagocytosis, and prolongs their systemic circulation. It also increases PER effect for the long circulating NPs, providing unique opportunities for interaction with cell surfaces (Babu et al., 2013). These advantages of NPs' PEGylation increase

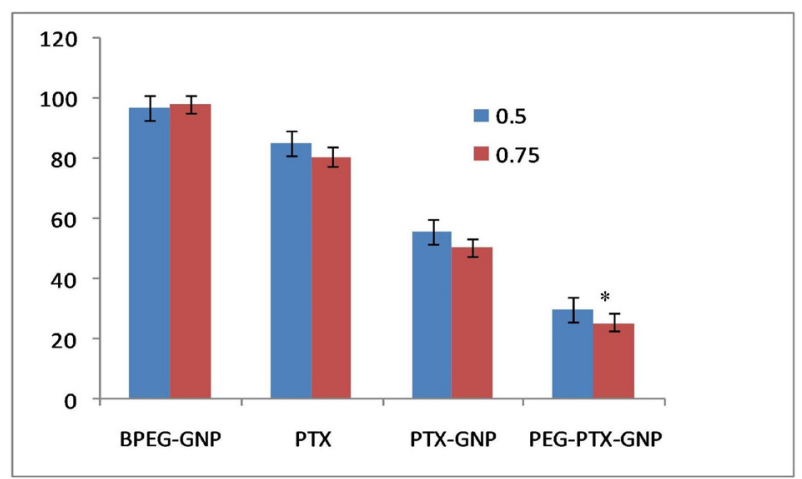

Figure 3. Comparative cytotoxicity of blank PEGylated gelatin NPs (BPEG-GNP), pure PTX (PTX), PTX-loaded gelatin NPs (PTXGNP) and PEGylated gelatin PTX-loaded NPs (PEG-PTX-GNP) in L132 human lung cancer cell line at 0.5 and $0.75 \mathrm{mg} / \mathrm{mL}$.

All formulations showed concentration dependent cytotoxicity and PEG-PTX-GNP showed minimum cytotoxicity i.e., maximum antiproliferative effect on cell line $\left({ }^{*} P<0.05\right)$. The significance between the various formulations was evaluated using SAS software. ${ }^{*} P<0.05$. Data presentation of mean S.D. of \pm 1.5 

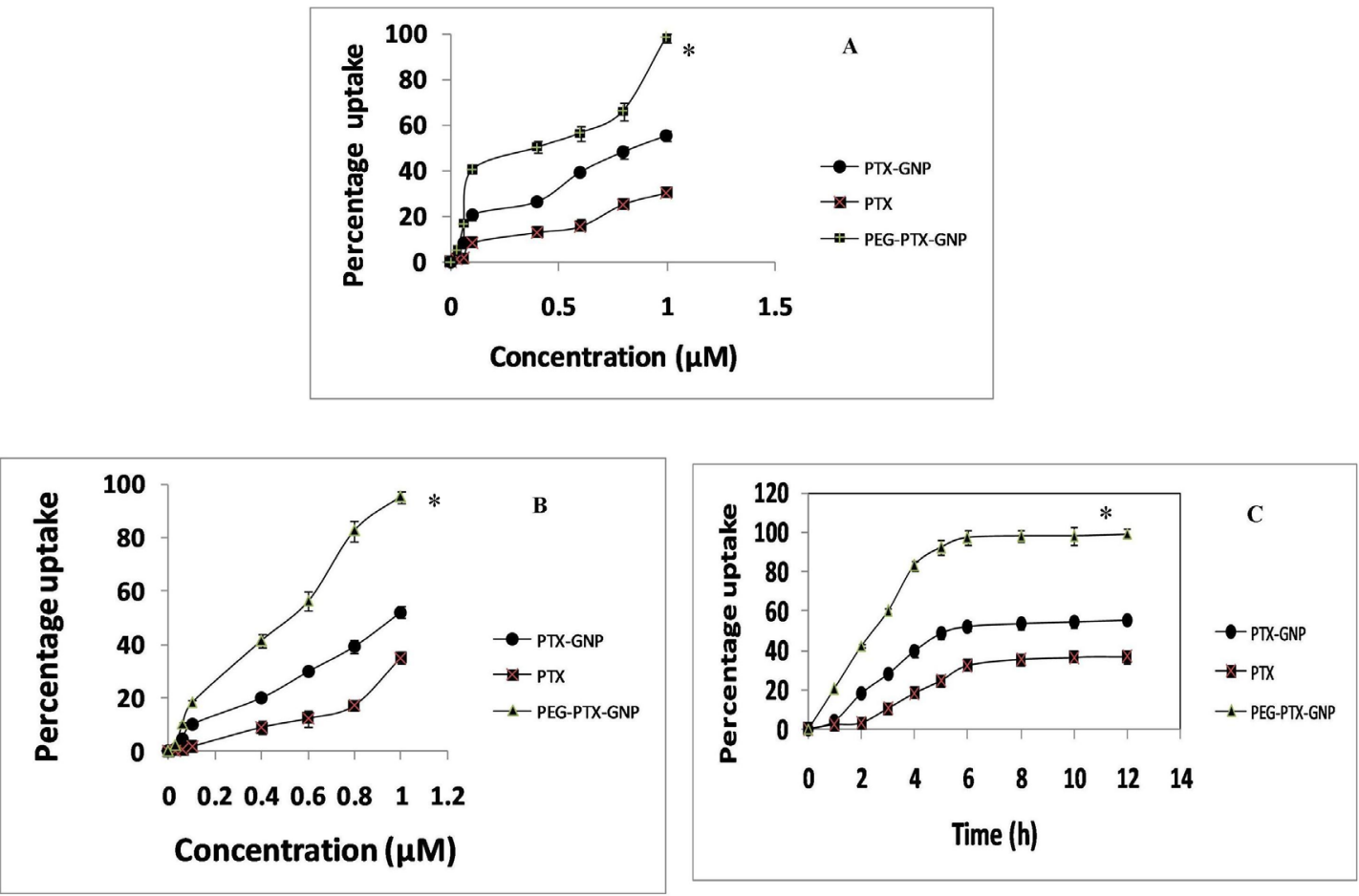

Figure 4. Comparative in vitro cellular uptake studies of pure PTX (PTX), PTX-loaded gelatin NPs (PTX-GNP) and PEGylated gelatin PTX-loaded NPs (PEG-PTX-GNP) in L132 cell line (A); in 293T cell line (B); averaged for both cell lines (C).

The significance of the difference between the various formulations was evaluated using SAS software. ${ }^{*} P<0.05$. $\left({ }^{*}\right.$ The results obtained in this study are statistically significant). Data presentation of mean S.D. of \pm 0.5

the cellular uptake of PEG-PTX-GNP when compared to PTX-GNP or pure PTX. The cellular uptake was found to be time dependent in all types of formulations in both types of cells. However, the saturation of cel- lular uptake was observed after $6 \mathrm{~h}$ of incubation with nanoparticles.
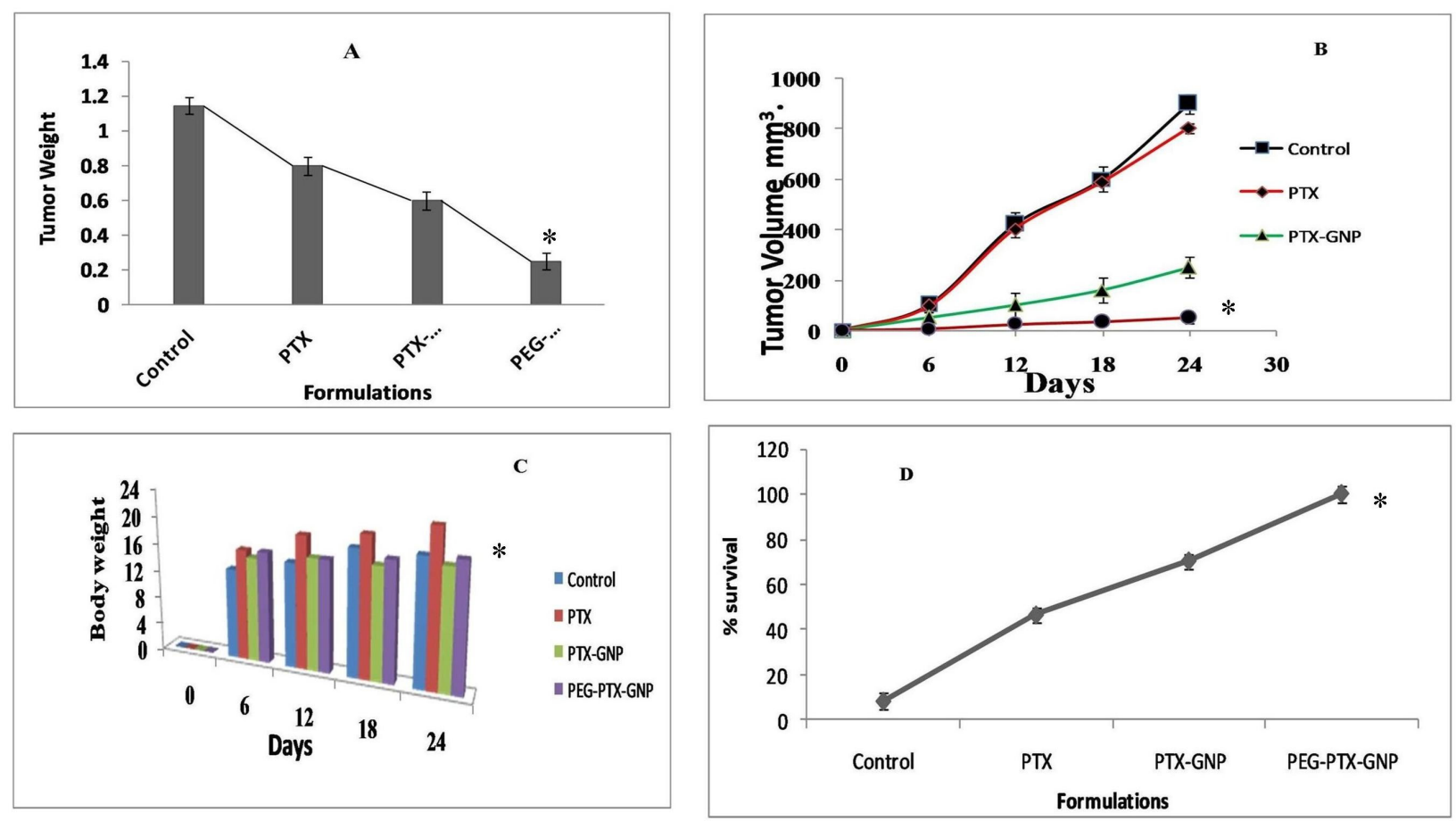

Figure 5. Tumor weight (A) and volume (B) suppression after treatment with formulations; Body weight (C) and survival rate (D) after treatment with formulations.

The significance of the differences between the various formulations was evaluated using SAS software. ${ }^{*} P<0.05\left({ }^{*}\right.$ The results obtained in this study are statistically significant). Data presentation of mean S.D. of \pm 0.5 


\section{In vivo antitumor activity}

Antitumor activity of the NPs was determined based on the measurements of tumor volume, tumor weight, body weight and survival rate of the BALB/c-nu/ nu athymic mice injected with L132 tumor cells. As shown in Fig. $5 \mathrm{~A}$, the smallest tumor in terms of weight $(0.25 \mathrm{~g})$ was observed in animals treated with PEGPTX-GNP, followed by PTX-GNP (0.6 g), PTX (0.8 g) and the control group, which showed the largest tumor weight of $1.15 \mathrm{~g}$. The smallest tumor growth in animals indicates the maximum antitumor activity of the drug and can be attributed to the higher internalization and accumulation of NPs in tumor tissue.

The control group showed the highest tumor volume (nearly $900 \mathrm{~mm}^{3}$ ), while the PEG-PTX-GNP treated group's mean tumor volume was a mere $50 \mathrm{~mm}^{3}$ and PTX-GNP treated group tumor volume was $250 \mathrm{~mm}^{3}$ as shown in Fig. 5B.

The PEG-PTX-GNP treated group showed nearly 18fold tumor suppression growth over the period of 24 days, as compared to the control group. This long-lasting tumor suppression was attributed to slow and gradual release of PTX from the PEGylated gelatin nanoparticles and enhanced cellular uptake and drug internalization in cancer tissue. In neither of the treated groups a reduction in body weight was observed, indicating that the administered PTX dose was well acceptable and tolerated (Fig. 5C).

The sustained and gradual PTX release form the NPs prevented accumulation and harm to the normal (non-cancer) cells. 24 days post-injection, the survival rate of animals in PEG-PTX-GNP treated group was a $100 \%$, proving the safety and efficacy of the formulation. The control group showed the lowest survival rate of just $8.5 \%$ at the end of study (Fig. 5 D). Overall, PEG-PTX-GNP showed the excellent antitumor activity.

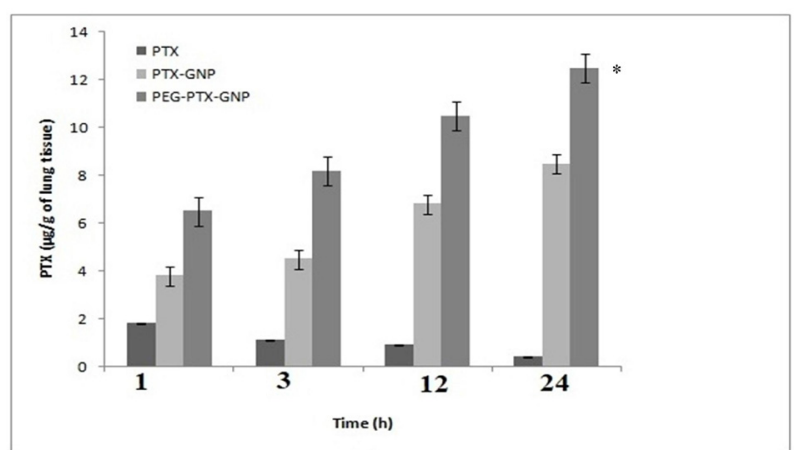

Figure 6. Pulmonary deposition of PTX at various time points. In case of PTX-GNP, the pulmonary deposition was increased from $3.8 \mu \mathrm{g} / \mathrm{g}$ to $8.5 \mu \mathrm{g} / \mathrm{g}$ over $24 \mathrm{~h}$ post-treatment. The pulmonary deposition rate was highest $(6.5$ to $12.55 \mu \mathrm{g} / \mathrm{g})$ for the PEG-PTXGNP formulations $\left({ }^{*} P<0.05\right)$. The significance between the various formulations was evaluated using SAS software. Data presentation of mean S.D. of \pm 0.5

\section{Pulmonary deposition study}

The pulmonary deposition of PTX was determined in the animals treated with pure PTX, PTX-GNP or PEGPTX-GNP. The mice were sacrificed at 1, 3, 12 and 24 $\mathrm{h}$ post-treatment. The maximum deposition associated with pure PTX was $1.85 \mu \mathrm{g} / \mathrm{g}$ after 1 hour, but later no increase in deposition was observed. Instead, pure PTX showed decline in pulmonary deposition over time. But in case of PTX-GNP and PEG-PTX-GNP formulations, a gradual increase in pulmonary deposition was observed over time. In case of PTX-GNP, the pulmonary deposition was increased from $3.8 \mu \mathrm{g} / \mathrm{g}$ to $8.5 \mu \mathrm{g} / \mathrm{g}$ within the period of 24 hours. The pulmonary deposition rate was the highest $(6.5$ to $12.55 \mu \mathrm{g} / \mathrm{g})$ for the PEG-PTX-GNP formulations (see Fig. 6). Maximum pulmonary deposition of PTX is desired for the best therapeutic effect for

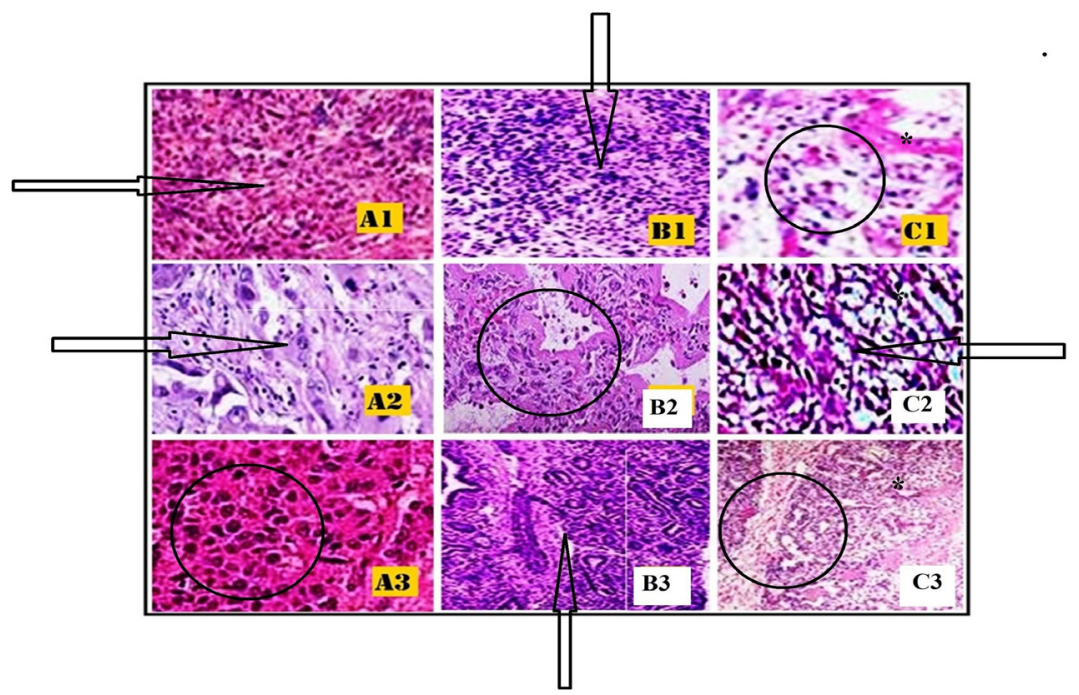

Figure 7. Histopathology and Immunohistochemical analysis:

(A1): Histopathology of tumor sections using hematoxylin stain obtained from control mice (Denser mass observed control A1 group shown by arrow); (A2): Histopathology of tumor sections using hematoxylin stain obtained from control mice (Denser mass observed in control group A2 shown by arrow); (A3): Histopathology of tumor sections using hematoxylin stain obtained from control mice (Denser mass observed in control group A3 shown by circle; (B1): Histopathology of tumor sections using eosin stain obtained from mice treated with PTX-GNP (missing of denser and crowded mass shown by arrow); (B2): Histopathology of tumor sections using eosin stain obtained from mice treated with PTX-GNP (Missing of denser and crowded mass shown by circle); (B3): Histopathology of tumor sections using eosin stain obtained from mice treated with PTX-GNP (Missing of denser and crowded mass shown by arrow); (C1): Immunohistochemical analysis of cleaved caspase 3 from tumor section collected from mice treated with PEG-PTX-GNP (missing of denser and crowded mass shown by circle); (C2): Immunohistochemical analysis of cleaved caspase 3 from tumor section collected from mice treated with PEG-PTX-GNP (Missing of denser and crowded mass shown by circle); (C3): Immunohistochemical analysis of cleaved caspase 3 from tumor section collected from mice treated with PEG-PTX-GNP (missing of denser and crowded mass shown by circle). The significance between the various formulations was evaluated using SAS software. $\left({ }^{*} P<0.05\right)$. 
the treatment of NSCLC. The continuous increase in pulmonary deposition of PEG-PTX-GNP over time was the result of PEGylation and smaller particle size of the formulations (Sukumar et al., 2013).

\section{Histopathology and immunohistochemistry of the tumor}

A dense and crowded extracellular mass was observed in the tumors from the control animals, while it was missing in animals treated with either PTX-GNP or PEG-PTX-GNP formulations, which indicated the anticancer potential of the formulations (Fig. 7A).

The immunohistochemical analysis also confirmed the antitumor potential of the PTX-GNP and PEGPTX-GNP formulations. The highest capase-3 levels were observed in the tumor specimens from the PEGPTX-GNP-treated animals. Lower caspase-3 levels were observed in PTX-GNP-treated group. These observations indicate a greater anticancer potential of PEGPTX-GNP. Conversely, MMP-9 expression was significantly reduced in animals treated with PEG-PTX-GNP as compared to PTX-GNP treatment group. Therefore, it could be concluded that PEG-PTX-GNP had greater anticancer potential than other tested formulations. We propose that it was due to the PEGylation of NPs, which increased cellular uptake and internalization in cancer tissue.

\section{CONCLUSION}

The PEGylated NPs showed a gradual and slowrelease profile upon systemic administration, enhanced encapsulation efficiencies, lower particle size, significant cytotoxicity in L132 and 293T cell lines and an increased pulmonary tissue deposition and antitumor activity and lesser cytotoxicity than non-PEGylated NPs. This study supports the potential use of paclitaxel loaded PEGylated gelatin targeted nanoparticles for improved efficacy in non-small cell lung cancer (NSCLC).

\section{Declaration of Conflict}

The authors declare no conflict of interest for the presented manuscript.

\section{Funding statement}

This research was funded by Nanjing Hope Medical Laboratory Co., Ltd (NO: 2020HP1070).

\section{REFERENCES}

Ahmad I, Longenecker M, Samuel J, Allen TM (1993) Antibodyta,rgeted delivery of doxorubicin entrapped in sterically stabilized liposomes can eradicate lung cancer in mice. Cancer Res 53: 1484 1488

Amjadi S, Hamishehkar H, Ghorbani M (2019) A novel smart PEGylated gelatin nanoparticle for co-delivery of doxorubicin and betanin: A strategy for enhancing the therapeutic efficacy of chemotherapy. Mat Sci Engin C 97: 833-841. https://doi.org/10.1016/j. msec.2018.12.104

Babu A, Templeton AK, Munshi A, Ramesh R (2013). Nanoparticlebased drug delivery for therapy of lung cancer: progress and challenges. J Nanomat 1: 1-11. https://doi.org/10.1155/2013/863951

Du WL, Niu SS, Xu YL, Xu ZR, Fan CL (2009). Antibacterial activity of chitosan tripolyphosphate nanoparticles loaded with various metal ions. Carbobydr Poly 75: 385-389. https://doi.org/10.1016/j. carbpol.2008.07.039

Garon EB, Rizvi NA, Hui R (2015) Pembrolizumab for the treatment of non-small-cell lung cancer. New Engl J Med 372: 2018-2028. https://doi.org/10.1056/NEJMoa1501824

Goncalves A, Braguer D, Kamath K, Martello L, Briand C, Horwitz S, Wilson L, Jordan MA (2001) Resistance to Taxol in lung cancer cells associated with increased microtubule dynamics. Proc Natl Acad Sci 98: 11737-11742. https://doi.org/10.1073/pnas.191388598

Hamarat Sanlier S, Yasa M, Cihnioglu AO, Abdulhayoglu M, Yilmaz H, Ak G (2016) Development of gemcitabine-adsorbed magnetic gelatin nanoparticles for targeted drug delivery in lung cancer. Art Cells Nanomed Biotechnol 44: 943-949. https://doi.org/10.3109/2169 1401.2014.1001493

Hao N, Liu H, Li L, Chen D, Li L, Tang F. (2012) In vitro degradation behavior of silica nanoparticles under physiological conditions. I Nanosci Nanotechnol 12: 6346-6354. https://doi.org/10.1166/ inn.2012.6199

Karthikeyan S, Prasad NR, Ganamani A, Balamurugan E (2013) Anticancer activity of resveratrol-loaded gelatin nanoparticles on NCIH460 non-small cell lung cancer cells. Biomed Prevent Nutrit 3: 64-73. https://doi.org/10.1016/j.bionut.2012.10.009

Kim I, Byeon HJ, Kim TH (2012) Doxorubicin-loaded highly porous large PLGA microparticles as a sustained-release inhalation system for the treatment of metastatic lung cancer. Biomaterials 33: 5574 5583. https://doi.org/10.1016/j.biomaterials.2012.04.018

Li K, Liang N, Yang H, Liu H, Li S (2017) Temozolomide encapsulated and folic acid decorated chitosan nanoparticles for lung tumor targeting: improving therapeutic efficacy both in vitro and in vivo. Oncotarget 8: 111318. https://doi.org/10.18632/oncotarget.22791

Liu J, Liu J, Chu L, Wang Y, Duan Y, Feng L, Yang C, Wang L, Kong D (2011) Novel peptide-dendrimer conjugates as drug carriers for targeting non-small cell lung cancer. Int J Nanomed 6: 59-69. https://doi.org/10.2147/IJN.S14601

Long JT, Cheang TY, Zhuo SY, Zeng RF, Dai QS, Li HP, Fang S (2014) Anticancer drug-loaded multifunctional nanoparticles to enhance the chemotherapeutic efficacy in lung cancer metastasis. J Nanobiotechnol 12: 1-11. https://doi.org/10.1186/s12951-014-0037-5

Lu Z, Yeh TK, Tsai M, Au JL, Wientjes MG (2004) Paclitaxel-loaded gelatin nanoparticles for intravesical bladder cancer therapy. Clin Cancer Res 10: 7677-7684. https://doi.org/10.1158/1078-0432.CCR04-1443

Lv S, Tang Z, Li M (2014) Co-delivery of doxorubicin and paclitaxel by PEG-polypeptide nano vehicle for the treatment of non-small cell lung cancer. Biomaterials 35: 6118-6129. https://doi.org/10.1016/j. biomaterials.2014.04.034

Mehrotra A, Nagarwal RC, Pandit JK (2011) Lomustine loaded chitosan nanoparticles: characterization and in-vitro cytotoxicity on human lung cancer cell line L132. Chem Pharm Bull 59: 315-320. https://doi.org/10.1248/cpb.59.315

Peng G, Tisch U, Adams O (2009) Diagnosing lung cancer in exhaled breath using gold nanoparticles. Nat Nanotechnol 4: 669-673. https://doi.org/10.1038/nnano.2009.235

Pless M, Stupp R, Ris HB (2015) Induction chemo radiation in stage IIIA/N2 non-small-cell lung cancer: a phase 3 randomised trial. Lancet 386: 1049-1056. https://doi.org/10.1016/S01406736(15)60294-X

Schädlich A, Caysa H, Mueller T, Tenambergen F, Rose C, Göpferich A, Kuntsche J, Mäder K (2015) Tumor accumulation of NIR fluorescent PEG-PLA nanoparticles: Impact of particle size and human xenograft tumor model. ACS Nano 5: 8710-8720. https://doi. org/10.1021/nn2026353

Scheeren LE, Nogueira DR, Macedo LB, Vinardell MP, Mitjans M, Infante MR, Rolim CM (2016) PEGylated and poloxamer-modified chitosan nanoparticles incorporating a lysine-based surfactant for $\mathrm{pH}$-triggered doxorubicin release. Colloids and Surfaces B: Biointerfaces 138: $117-127$

Scott WJ, Howington J, Feigenberg S (2007) Treatment of non-small cell lung cancer stage I and stage II: ACCP evidence-based clinical practice guidelines. Chest 132: 234S-2342S. https://doi.org/10.1378/ chest.07-1378

Shen J, Yin Q, Chen L, Zhang Z, Li Y (2012) Co-delivery of paclitaxel and survivin shRNA by pluronic P85-PEI/TPGS complex nanoparticles to overcome drug resistance in lung cancer. Biomaterials 33: 8613-8624. https://doi.org/10.1016/j.biomaterials

Singh P, Gupta U, Asthana A, Jain NK (2008) Folate and folatePEG-PAMAM Dendrimers: synthesis, characterization, and targeted anticancer drug delivery potential in tumor bearing mice. Bioconjugate Chem 19: 2239-2252. https://doi.org/10.1021/bc800125u

Su WP, Cheng FY, Shieh DB, Yeh CS, Su WC (2012) PLGA nanoparticles codeliver paclitaxel and Stat3 siRNA to overcome cellular resistance in lung cancer cells. Int J Nanomed 7: 4269. https://doi. org/10.2147/IJN.S33666

Sukumar UK, Bhushan B, Dubey P, Matai I, Sachdev A, Packirisamy G (2013). Emerging applications of nanoparticles for lung cancer diagnosis and therapy. Int Nano Lett 3: 45. https://doi. org/10.1186/2228-5326-3-45

Tahara Y, Yoshikawa T, Sato H (2017) Encapsulation of a nitric oxide donor into a liposome to boost the enhanced permeation and retention (EPR) effect. Med Chem Comm 8: 415-421. https://doi. org/10.1039/C6MD00614K

Torre LA, Siegel RL, Jemal A (2016) Lung cancer statistics. Lung cancer and personalized medicine. pp 1-19. Springer Cham 
Tran PH, Tran TT, Lee BJ (2014) Biodistribution and pharmacokinetics in rats and antitumor effect in various types of tumor-bearing mice of novel self-assembled gelatine-oleic acid nanoparticles containing paclitaxel. I Biomed Nanotechnol 10: 154-165. https://doi. org/10.1166/jbn.2014.1660

Tseng CL, Wu SY, Wang WH (2008). Targeting efficiency and biodistribution of biotinylated-EGF-conjugated gelatin nanoparticles administered via aerosol delivery in nude mice with lung cancer. Biomaterials 29: 3014-3022. https://doi.org/10.1016/j.biomaterials.2008.03.033
Yoo MK, Park IK, Lim HT (2012) Folate-PEG-superparamagnetic iron oxide nanoparticles for lung cancer imaging. Acta Biomaterialia 8: 3005-3013. https://doi.org/10.1016/j.actbio.2012.04.029

Zhang X, Gan Y, Gan L, Nie S, Pan W (2008). PEGylated nanostructured lipid carriers loaded with 10 hydroxycamptothecin: an efficient carrier with enhanced anti tumour effects against lung cancer. J Phar Pharmacol 60: 1077-1087. https://doi.org/10.1211/jpp.60.8.0014. 